CIC. Cuadernos de Información y Comunicación ISSN: 1135-7791

http://dx.doi.org/10.5209/CIYC.60910

\title{
Condiciones y rendimientos de la Investigación en Comunicación: la visión de los académicos
}

\author{
Juan Antonio Gaitán ${ }^{1}$; Carmen Caffarel$^{2}$; Carlos Lozano ${ }^{3}$; José Luis Piñuel ${ }^{4}$
}

Recibido: 13 de marzo de 2018 / Aceptado: 3 de julio de 2018

Resumen. El Proyecto MapCom (www.mapcom.es) lanzó en 2017 una encuesta destinada al universo de investigadores en Comunicación censados como doctores en nómina en los centros universitarios españoles con grados y postgrados en las carreras de Comunicación, en sociedades científicas y en programas de doctorado, solicitándoles vía internet cumplimentar un formulario centrado en su experiencia investigadora. En este artículo se ofrecen los datos sobre las condiciones y rendimientos de su actividad Investigadora y de su estatus como investigador, obtenidos en aquella encuesta. Se preguntó sobre cuál era su valoración sobre las condiciones materiales (financiación, becas y contratos, burocracia, instalaciones), organizacionales (requisitos de las convocatorias, formación metodológica, redes científicas, capital humano) e institucionales (compatibilidad con la docencia, relación Universidad/ empresa, Agencias de Evaluación, Congresos, Revistas). De estos datos se desprende la tendencia a valorar negativamente las condiciones materiales, mientras que las condiciones organizacionales e institucionales recibieron valoraciones positivas y negativas diversas según los ítems. Los rendimientos recibieron valoraciones más positivas cuando se relacionaban con el corporativismo académico que cuando se vinculaban a la competencia científica.

Palabras clave: Encuesta MapCom, Censo investigadores, Universidad española, Investigación Comunicación.

\section{[en] Conditions and performances of the Research in Communication: the vision of the academics}

\begin{abstract}
The MapCom Project (www.mapcom.es) launched a survey in 2017 aimed at researchers in the field of communication who were under contract as Doctors at Spanish universities with graduate or postgraduate Degrees in Communication, or research associations and doctorate programmes. It asked respondents to fill in an online form based on their research experience. In this article we present the data concerning the conditions and performance of the research activity and their status as researchers, obtained from that survey. Interviewees were asked how they rated the working conditions both in terms of material (funding, grants, contracts, red-tape, facilities), organisation (requirements of the call, teaching methodology, scientific networks, human capital) and institutions (compatibility with lecturing, relations between the unversity and the business world. Assessment Agencies, Congresses
\end{abstract}

$1 \quad$ Universidad Complutense de Madrid. España.

jagamo@ucm.es

2 Universidad Rey Juan Carlos. España.

carmen.caffarel@urjc.es

3 Universidad Rey Juan Carlos. España.

carlos.lozano@urjc.es

$4 \quad$ Universidad Complutense de Madrid. España.

pinuel@ucm.es 
and Journals). The conclusion was that there was a tendency to have a negative evaluation of materials whereas, in terms of the organisation and institution, the evaluation was a mixture of positive and negative depending on the item considered. The evaluation of performance related to academic corporativism tended to be more positive than those related to scientific competencies.

Keywords: MapCom Survey, Census of Researchers, University in Spain, Communication Research.

Sumario. Introducción. La Encuesta MapCom. Contextualización y metodología. El formulario de encuesta. Resultados y discusión. a) Valoración de las condiciones materiales, organizacionales, e institucionales según la experiencia de los encuestados. b) La valoración de la experiencia investigadora en relación al beneficio obtenido como retorno. Discusión y Conclusiones. Bibliografía.

Cómo citar: Gaitán, J. A.; Caffarel, C.; Lozano, C.; Piñuel, J. L. (2018). Condiciones y rendimientos de la Investigación en Comunicación: la visión de los académicos, en CIC. Cuadernos de Información y Comunicación 23, 105-123.

\section{Introducción}

La investigación sobre condiciones y rendimientos de la Investigación en Comunicación, forma parte de un objeto de estudio más amplio, como es el estudio de las prácticas y metodologías de investigación en Comunicación. En nuestro contexto cultural se remontan a finales del siglo XX auspiciadas muchas de ellas por la AICE (Asociación de Investigadores en Comunicación del Estado Español) y antecedente de la actual AE-IC (Asociación Española de Investigación en Comunicación). Autores como Caffarel, Domínguez y Romano (1989), Caffarel y Cáceres (1993), y Jones (1994, 1998 y 2000) al llegar el vigésimo aniversario de la creación de las primeras Facultades de Ciencias de la Información en España, analizaron el estado de la cuestión de la investigación en Comunicación. Posteriormente cabe destacar a RodrigoAlsina y García-Jiménez (2010), Arcila-Calderón, Piñuel-Raigada y Calderín Cruz (2013). En la dimensión bibliométrica de las tesis doctorales en España merecen mención especial los trabajos de Delgado López-Cózar et al. (2006), Castillo y Xifra (2006), Fuentes Pujol y Arguimbau-Vivó (2010), Repiso et al. (2011) y más recientemente Blázquez-Ochando (2015). Sobre la investigación en publicaciones científicas, cabe destacar los trabajos de Fernández-Quijada y Masip (2013), Martínez-Nicolás y Saperas-Lapiedra (2009, 2011), y López Rabadán y Vicente-Mariño (2011), Piñuel-Raigada (2011); Lozano-Ascencio y Gaitán-Moya (2011); y Piñuel-Raigada, Lozano-Ascencio y García-Jiménez (eds.) (2011).

Ahora bien, en este marco del estudio de las prácticas y metodologías de investigación en Comunicación, nunca se ha abordado, como objeto particular de estudio, las condiciones y rendimientos de la Investigación en Comunicación y tampoco desde la experiencia que sobre ello manifiestan los investigadores.

En el año 2013, en la convocatoria estatal de Proyectos I+D, cuatro equipos coordinados, uno como equipo coordinador con sede en la Universidad Complutense (UCM) y los otros tres en la Universidad del País Vasco (EHU), la Universitat Jaume I (UJI), y la Universidad de Málaga (UMA) presentaron solicitud para realizar un estudio con el título "El sistema de investigación en España sobre prácticas sociales de Comunicación. Mapa de Proyectos, Grupos, Líneas, Objetos de estudio y Métodos", cuyo acrónimo adoptado fue MAPCOM (ref. CSO2013-47933-C4). El Proyecto MapCom, aspiraba a contribuir a la elaboración de un mapa capaz de represen- 
tar el desarrollo del sistema de investigación en España sobre prácticas sociales de Comunicación, estableciendo:

$1^{\text {o: }}$ Un banco de datos de acceso abierto sobre Proyectos, Grupos, Líneas, Objetos de estudio y Métodos de investigación, recopilando y analizando las memorias científicas de las solicitudes y de los informes de todos aquellos proyectos de investigación financiados desde 2007 por convocatorias nacionales, así como de las Tesis Doctorales aprobadas también desde 2007, en las universidades españolas con titulaciones de grado y postgrado en las áreas de la Comunicación, según zonas geográficas en que operativamente se distribuyeron el trabajo los equipos de investigación coordinados de la siguiente manera: Área 1: Castilla y León, Castilla La Mancha, Madrid y La Rioja; Area 2: Galicia, País Vasco, Navarra y Aragón; Área 3: Andalucía, Extremadura y Canarias; y Área 4: Cataluña, Comunidad Valenciana y Murcia.

Los informes de esta fase se pueden consultar en la web de MapCom http://www. mapcom.es/investigacion/fase-1 así como sus bases de datos en http://www.mapcom.es/bases-de-datos . Y entre las publicaciones a que se ha dado lugar destacan Caffarel, C. Izquierdo, P. y Núñez, S.: (2018); Martín Algarra, M.; Serrano-Puche, J. y Rebolledo, M. (2018); Caffarel, C, y Ortega, F; Gaitán, J:A: (2017), Díaz Nosty, B. y de Frutos, R. (coord.) (2016), Gaitán Moya, J.A.; Lozano Ascencio, C. y Piñuel Raigada, J.L. (2016), Gaitán, J. A., Lozano, C., Piñuel, J. L. (2016), Lozano Ascencio, C y Piñuel Raigada, JL. (2015), Piñuel, J. L., Gaitán, J. A., Lozano, C., Gallardo, L. (2017) Piñuel, J. L., Lozano, C., Gaitán, J. A. (2015), Barranquero Carretero, A, y Limón Serrano, N. (2017), López-Escobar, E. y Martín Algarra, M. (2016).

$2^{\circ}$ : En segundo lugar, estableciendo el discurso hegemónico de los académicos que, en una segunda fase del proyecto, participaron en debates sobre resultados obtenidos del análisis de Tesis y Proyectos I+D. Fueron convocados a debate investigadores principales (IP) y directores de tesis doctorales, con responsables de grupos consolidados de investigación, con gestores académicos de proyectos y programas de investigación, con portavoces de sociedades científicas, con editores de revistas fuente del ámbito de la Comunicación y, finalmente, con investigadores junior, en sesiones celebradas en Madrid, Barcelona y Málaga utilizando la técnica conocida como Phillips 66. Se pueden consultar los informes de estos debates en http:/www.mapcom.es/investigacion/ fase-2. Y entre las publicaciones a que se ha dado lugar destacan Peñafiel, C., Torres, E., e Izquierdo, P., (2017); Ortega, F., Azurmendi, A. y Muñoz Saldaña, M. (2018).

$3^{\circ}$ : Una tercera fase consistió en lanzar un formulario de encuesta aplicado online al universo de investigadores del territorio español, censados tanto en sociedades científicas como en registros académicos de las universidades. Se pueden consultar los informes de estos debates en http://www.mapcom.es/investigacion/fase-3 . Y entre las publicaciones a que se ha dado lugar destacan Caffarel, C., Gaitán, J.A., Lozano, C. y Piñuel, J.L. (2018). En este artículo abordamos la explotación de un aspecto parcial de los resultados de esta encuesta: la visión de los académicos sobre condiciones y rendimientos de la Investigación en Comunicación.

\section{La Encuesta MapCom}

La encuesta se llevó a cabo entre mayo y agosto de 201. Se contactó vía correo electrónico con cerca de 2500 investigadores de la comunicación, censados como docto- 
res y estudiantes de doctorado en centros universitarios españoles con titulaciones de Grado y Postgrado en las carreras de Comunicación.

Accedieron al formulario de esta encuesta 1.254 investigadores, de los cuales 838 (el 34\% del censo) respondieron a las preguntas, finalizando el formulario 496 y respondiéndolo parcialmente 342 . Por último, 416 abandonaron sin contestar ninguna pregunta.

Las respuestas relacionadas con la identidad profesional y académica tuvieron que ver con la experiencia investigadora y sus reconocimientos: integración en grupos consolidados y en equipos de proyectos, sexenios, pertenencia a sociedades científicas, registro en bases de datos de investigación, etc. Las contestaciones relacionadas con la apreciación de su propia actividad investigadora destacan por denunciar mayoritariamente el escaso reconocimiento de la dedicación a investigar frente a la dedicación docente, cuando de forma paradójica es el resultado de la investigación del que se hace depender las evaluaciones de la carrera académica y de la calidad de las titulaciones que se imparten. El lector interesado en detalle por todos estos datos, puede consultarlos en http://www.mapcom.es/investigacion/fase-3. En este artículo nos limitamos a exponer y comentar los datos referidos a las Condiciones y rendimientos de la actividad investigadora en Comunicación.

\section{Contextualización y metodología}

Por lo que respecta al universo de destinatarios de la encuesta, se decidió tomar en consideración concretamente:

a) Investigadores censados en Facultades de Comunicación en España (todos los doctores en nómina de cada universidad)

b) Investigadores miembros de Sociedades científicas (miembros doctores que no estén en nómina en ninguna universidad)

c) Investigadores en formación en los programas de Doctorado.

El universo del primer grupo está conformado por todos los profesores doctores en las Facultades con estudios de grado universitario en Comunicación, lo que comprendía todas las universidades incluidas en el universo de tesis doctorales y proyectos $\mathrm{I}+\mathrm{D}$ de la primera fase de MapCom. El segundo grupo contemplaba a los doctores que no imparten docencia universitaria, pero que sí pertenecen a alguna sociedad científica, desde las cuales se facilitó el acceso a la encuesta. Respecto al tercer grupo de investigadores en formación en los programas de Doctorado, los equipos de las Áreas MapCom contactaron con los Coordinadores de los programas de Doctorado para que utilizaran sus censos y enviaran a los doctorandos de último curso, el enlace de la encuesta.

Tras elaborar el censo correspondiente a los universos citados, se cursaron un total de 2.418 invitaciones personalizadas a investigadores adscritos a universidades españolas, así como una invitación abierta indeterminada a través de sociedades científicas y programas de doctorado. Se obtuvieron 838 contestaciones para una tasa de respuesta efectiva de $34,66 \%$. Para estimar la representatividad por estratos de la muestra obtenida, se tomaron en cuenta las variables de Comunidad Autónoma (Tabla 1) y universidad, a las que pertenecía el investigador. Específicamente, se 
estimaron los porcentajes esperados mínimos y máximos para cada categoría de las variables, con el fin de comprobar que ninguna de las Comunidades Autónomas (CC. AA.) y universidades quedaran infra o supra representadas en la muestra final con el sesgo de auto-respuesta. Para ello se calculó en el censo la media y la desviación típica de cada sub-categoría (utilizando variable dummy para cada valor), obteniendo así intervalos de confianza (\%min.= M-DT y \%máx.=M+DT). Como se observa en la Tabla 1, los porcentajes de cada CC.AA. encajaron dentro de los límites esperados, por lo que así los estratos en la muestra resultaron correctamente representados con respectos a los del censo. También encajaron dentro de los límites esperados los

Tabla 1. Distribución de investigadores invitados y proporción de respuestas por Comunidad Autónoma y con porcentajes esperados mínimos y máximos para cada estrato.

\begin{tabular}{|c|c|c|c|c|c|c|}
\hline CC.AA. & Invitados & $\%$ & Muestra & $\%$ Muestra & $\begin{array}{c}\text { \% Min } \\
\text { Esperado }\end{array}$ & $\begin{array}{c}\text { \% Máx } \\
\text { Esperado }\end{array}$ \\
\hline Andalucía & 253 & 10,5 & 77 & $9,20 \%$ & $-20,15 \%$ & $41,07 \%$ \\
\hline Aragón & 43 & 1,8 & 19 & $2,30 \%$ & $-11,44 \%$ & $15,00 \%$ \\
\hline Asturias & 0 & 0 & 1 & $0,10 \%$ & $0,00 \%$ & $0,00 \%$ \\
\hline Baleares & 0 & 0 & 7 & $0,80 \%$ & $0,00 \%$ & $0,00 \%$ \\
\hline Canarias & 15 & 0,6 & 2 & $0,20 \%$ & $-7,23 \%$ & $8,47 \%$ \\
\hline Cantabria & 0 & 0 & 0 & $0,00 \%$ & $0,00 \%$ & $0,00 \%$ \\
\hline Castilla La Mancha & 20 & 0,8 & 4 & $0,50 \%$ & $-8,23 \%$ & $9,89 \%$ \\
\hline Castilla y León & 121 & 5 & 61 & $7,30 \%$ & $-16,81 \%$ & $26,81 \%$ \\
\hline Cataluña & 405 & 16,7 & 94 & $11,20 \%$ & $-20,60 \%$ & $54,10 \%$ \\
\hline Extremadura & 36 & 1,5 & 7 & $0,80 \%$ & $-10,62 \%$ & $13,60 \%$ \\
\hline Galicia & 110 & 4,5 & 39 & $4,70 \%$ & $-16,29 \%$ & $25,39 \%$ \\
\hline La Rioja & 26 & 1,1 & 13 & $1,60 \%$ & $-9,24 \%$ & $11,40 \%$ \\
\hline Madrid & 770 & 31,8 & 146 & $17,40 \%$ & $-14,76 \%$ & $78,44 \%$ \\
\hline Murcia & 84 & 3,5 & 32 & $3,80 \%$ & $-14,85 \%$ & $21,79 \%$ \\
\hline Navarra & 55 & 2,3 & 30 & $3,60 \%$ & $-12,64 \%$ & $17,18 \%$ \\
\hline País Vasco & 190 & 7,9 & 35 & $4,20 \%$ & $-19,05 \%$ & $34,77 \%$ \\
\hline Valencia & 290 & 12 & 79 & $9,40 \%$ & $-20,51 \%$ & $44,49 \%$ \\
\hline Fuera de España & & & 5 & $0,60 \%$ & & \\
\hline Sin contestación & & & 187 & $22,30 \%$ & & \\
\hline Total & 2418 & 100 & 838 & $100,00 \%$ & & \\
\hline
\end{tabular}

Fuente: Proyecto MapCom http://www.mapcom.es/media/files/Los_investigadores_de_la_comunicacion_en_Espana_ante_el_espejo. Encuesta_MapCom.pdf 
porcentajes de cada universidad, pero evitamos ofrecer la tabla porque obviamos exponer datos de las universidades al objeto de no alargar demasiado esta exposición. El lector interesado puede acudir a la Web citada de MapCom.

En resumen, de los 2418 invitados a contestar la encuesta mediante e-mail personalizado y la entrega de una contraseña para abrir el formulario, accedieron al cuestionario 1.254 investigadores, de los cuales 838 (el 34\% del censo) respondieron a las preguntas, finalizando el formulario 496 y respondiéndolo parcialmente 342 . Por último, 416 abandonaron sin contestar ninguna pregunta.

\section{El formulario de encuesta}

Con el propósito de conocer los perfiles de la identidad académica de los investigadores y las valoraciones de su experiencia en la investigación desarrollada en el campo de la comunicación, el cuestionario comprendió los apartados generales siguientes:

a) Información previa del encuestado sobre el Proyecto MapCom, al objeto de estimar si el interés mostrado al contestar la encuesta se podría deber al conocimiento previo sobre este proyecto.

b) Perfil como investigador (antigüedad, proyectos realizados, registros en bases de datos, sexenios, etc.) para poder relacionarlo con las respuestas que posteriormente se ofrecen al ser preguntado sobre su experiencia investigadora.

c) Adscripción profesional como investigador para relacionar su perfil de investigador con los datos del censo de investigadores (universidad de adscripción, rango académico/laboral, grupos/equipos de investigación, Comunidad Autónoma, Docencia ejercida, etc.).

d) Experiencia investigadora, para recabar información referida a la experiencia personal en la actividad investigadora desarrollada, tomando en consideración la fijación de objetivos, la elección de objetos de estudio, el tipo de técnicas empleadas en la elaboración y registro de datos, y la valoración de esta experiencia referida al beneficio obtenido como retorno.

e) Debilidades y Fortalezas de la Investigación en Comunicación, al valorar negativamente o positivamente las condiciones materiales, organizacionales, e institucionales a que según su experiencia ha estado sometida en España la actividad investigadora.

f) Inserción en los perfiles sociodemográficos del censo de investigadores en comunicación, respondiendo a las preguntas sobre edad, género, formación de origen y rango de ingresos económicos por la actividad profesional.

Con los datos obtenidos de esta encuesta, de los cuales aquí nos limitaremos a exponer y comentar los que corresponden a apartado e) sobre Debilidades y Fortalezas de la Investigación en Comunicación, al valorar negativa o positivamente las condiciones materiales, organizacionales, e institucionales a que según su experiencia ha estado sometida en España la actividad investigadora. La participación constatada al comprobar los estratos de la muestra por universidades y CC.AA respecto al censo, nos permite interpretar los datos obtenidos sabiendo que éstos son repre- 
sentativos del universo de investigadores de la comunicación en la universidad española con titulaciones en Comunicación.

\section{Resultados y discusión}

\section{a) Valoración de las condiciones materiales, organizacionales, e institucionales según la experiencia de los encuestados}

Se preguntó a los encuestados su valoración a propósito de las debilidades y fortalezas de la Investigación en Comunicación, al calificar negativa o positivamente las condiciones materiales, organizacionales, e institucionales a las que, según su experiencia, ha estado sometida en España la actividad investigadora. Para ello el formulario invitaba a puntuar cada ítem conforme a una escala de Likert, desde -3 como extremadamente negativo, hasta +3 , como extremadamente positivo. Las Tablas 2, 3, 4 y 5 permiten contemplar la comparación de valoraciones por Item.

A la vista de estos perfiles (Tabla 2), se puede colegir que para más de la mitad de los investigadores, pues contestan esta pregunta más del $50 \%$ de los encuestados, las condiciones materiales que acompañan a los procesos de investigación son valoradas negativamente, salvo al referirse a instalaciones y laboratorios, cuya valoración es en conjunto positiva. En cambio, la tendencia a la valoración negativa no es la dominante para los aspectos propuestos a ser juzgados en lo que respecta a condiciones

Tabla 2. Debilidades y fortalezas de la Investigación en Comunicación, al valorar las CONDICIONES MATERIALES.

\begin{tabular}{|c|c|c|c|c|c|}
\hline \multicolumn{3}{|c|}{ Subvenciones y financiación últimos 10 años } & \multicolumn{3}{|c|}{ Becas y Contratos últimos 10 años } \\
\hline Respuesta & $\begin{array}{l}\text { Porcentale } \\
\text {. }\end{array}$ & Cantidad & Respuesta & Porcentole & contitaod \\
\hline .3 & $27.118 \square$ & 119 & .3 & ${ }^{23.468} \square$ & 103 \\
\hline .2 & 1845\% $\square$ & 81 & .2 & $20968 \square$ & 92 \\
\hline .1 & 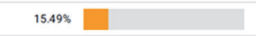 & 68 & .1 & ${ }_{14818}^{\square}$ & 65 \\
\hline o & 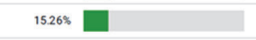 & 67 & - & ${ }^{14,355} \square$ & 63 \\
\hline+1 & ${ }^{14355} \square$ & 63 & +1 & 16.635 $\square$ & 73 \\
\hline+2 & 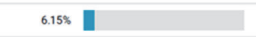 & 27 & +2 & 6.618 & 29 \\
\hline \multirow[t]{2}{*}{+3} & $3.199 x$ & 14 & +3 & 3.198 I & 14 \\
\hline & Total de respuestas & 439 & \multicolumn{3}{|c|}{ Total de respuestas } \\
\hline \multicolumn{3}{|c|}{ Trámites burocráticos últimos 10 años } & \multicolumn{3}{|c|}{ Instalaciones y laboratorios últimos 10 años } \\
\hline Respuesta & Porcentale & cantitad & $\begin{array}{ll}\text { Respuesta } \\
\end{array}$ & Porcentaje & Cantidad \\
\hline .3 & $34.78 \%$ & 152 & -3 & $17.41 \%$ & 74 \\
\hline-2 & ${ }_{17.39 \%}^{\square}$ & 76 & -2 & ${ }_{11298} \square$ & 48 \\
\hline-1 & 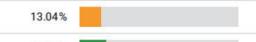 & 57 & -1 & $11.76 \%$ & 50 \\
\hline 0 & 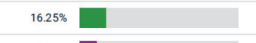 & 71 & 0 & $26.82 \%$ & 114 \\
\hline+1 & ${ }^{10.98 \%}$ 口 & 48 & +1 & ${ }^{18.82 \%} \square$ & 80 \\
\hline+2 & 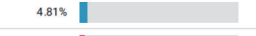 & ${ }^{21}$ & +2 & $8.71 \%$ & 37 \\
\hline \multirow[t]{2}{*}{+3} & 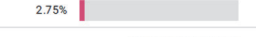 & 12 & +3 & $5.18 \% \mathbf{I}$ & 22 \\
\hline & Total de respuestas & ${ }^{437}$ & & & 425 \\
\hline
\end{tabular}

Fuente Proyecto MapCom http://www.mapcom.es/media/files/Los_investigadores_de_la_comunicacion_en_Espana_ante_el_espejo. Encuesta_MapCom.pdf 
Tabla 3. Debilidades y fortalezas de la Investigación en Comunicación, al valorar las CONDICIONES ORGANIZACIONALES.

\begin{tabular}{|c|c|c|c|c|c|}
\hline \multicolumn{3}{|c|}{ Requisitos exigidos en convocatorias públicas } & \multicolumn{3}{|c|}{ Programas de Formación metodológica } \\
\hline Respuesta & Porcentaje & cantidad & 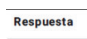 & Porcentale & Cantidad \\
\hline-3 & $18.52 \%$ & 80 & -3 & 20.70\% & ${ }^{89}$ \\
\hline-2 & $1921 \%$ & ${ }_{83}$ & .2 & ${ }_{16998} \square$ & 73 \\
\hline-1 & $19.68 \%$ & 85 & .1 & 15.81\% & 68 \\
\hline 0 & ${ }^{18775 \%}$ & ${ }^{81}$ & 0 & $25.12 \%$ & 108 \\
\hline+1 & ${ }^{15.51 \%}$ & 67 & +1 & ${ }_{14,42 \%}^{\square}$ & 62 \\
\hline+2 & 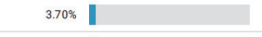 & 16 & +2 & ${ }_{4.42 \%}$ & 19 \\
\hline \multirow[t]{2}{*}{+3} & 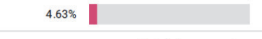 & ${ }^{20}$ & +3 & $2.56 \%$ I & 11 \\
\hline & Totalde respuestas & 432 & \multicolumn{3}{|c|}{ Total de respuest tas } \\
\hline \multicolumn{3}{|c|}{ Redes de cooperación y conocimiento } & \multicolumn{3}{|c|}{ Recursos humanos en los equipos } \\
\hline$\overline{\text { Respuesta }}$ & Porcentale & Cantidad & $\begin{array}{l}\text { Respuesta } \\
\text { a }\end{array}$ & Porcentaje & Cantidad \\
\hline-3 & $9.18 \% \square$ & 39 & .3 & $12.18 \% \square$ & 52 \\
\hline-2 & $7.53 \%$ & 32 & -2 & 10.30\% & 44 \\
\hline-1 & 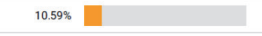 & 45 & -1 & 13.58\% & 58 \\
\hline 0 & 26.35\% & 112 & 0 & 26008 & 111 \\
\hline+1 & $29.18 \%$ & 124 & +1 & ${ }^{25068}$ & 107 \\
\hline+2 & ${ }^{14.128}$ & 60 & +2 & 9.138 & 39 \\
\hline \multirow[t]{2}{*}{+3} & ${ }^{3068}$ I & 13 & +3 & 3.758 | & 16 \\
\hline & Total de resppuestas & 425 & & & 427 \\
\hline
\end{tabular}

Fuente Proyecto MapCom

http://www.mapcom.es/media/files/Los_investigadores_de_la_comunicacion_en_Espana_ante_el_espejo.

Encuesta_MapCom.pdf

organizacionales de los procesos de investigación. Así, si las valoraciones positivas aparecen menos al juzgar los Requisitos exigidos en convocatorias públicas y los Programas de Formación metodológica, las valoraciones positivas aparecen en mayor medida cuando se aprecian las Redes de cooperación y conocimiento, y los Recursos humanos en los equipos.

Al apreciar las condiciones de los marcos institucionales referidos a la compatibilidad de investigación y docencia, a las relaciones entre Universidad y Empresa, o a las Agencias de Evaluación y a la calidad de Congresos y participación, aparecen (Tabla 4) mayoritariamente valoraciones positivas sólo al juzgar la calidad de Congresos y participación, mientras que son más frecuentes las valoraciones extremadamente negativas (-3) al juzgar la compatibilidad entre investigación y docencia, las relaciones entre Universidad y Empresa y al juzgar a las Agencias de evaluación ANECA, ANEP, y CNEAI.

Finalmente, aunque no es una condición institucional más, el rol que desempeñan las Revistas científicas y los efectos de su Indexación, al objeto de poder comparar el perfil de su valoración entre los encuestados con el perfil de la valoración de los otros marcos institucionales, se incluyó este ítem junto a los demás referidos a las condiciones institucionales; y este perfil aparece en la Tabla 5, donde vuelve a incluirse el perfil de la valoración de Congresos y participación, junto al de la valoración de las Revistas científicas y su indexación. 
Tabla 4. Debilidades y fortalezas de la Investigación en Comunicación, al valorar las CONDICIONES INSTITUCIONALES.

\begin{tabular}{|c|c|c|c|c|c|}
\hline \multicolumn{3}{|c|}{ Compatibilidad investigación y Docencia } & \multicolumn{3}{|c|}{ Relaciones entre Universidad y Empresa } \\
\hline Respuesta & Porcentaje & Cantidad & Respuesta & Porcentaje & Cantidad \\
\hline-3 & $29.68 \%$ & 130 & -3 & $24.88 \%$ & 107 \\
\hline-2 & $17.35 \%$ & 76 & -2 & $17.21 \%$ & 74 \\
\hline-1 & 12.33\% & 54 & -1 & $12.79 \%$ & 55 \\
\hline 0 & $16.67 \%$ & 73 & 0 & $23.72 \%$ & 102 \\
\hline+1 & $13.01 \%$ & 57 & +1 & $14.65 \%$ & 63 \\
\hline+2 & $7.08 \%$ & 31 & +2 & 5.35\% & 23 \\
\hline \multirow[t]{2}{*}{+3} & $3.88 \%$ & 17 & +3 & $1.40 \%$ | & 6 \\
\hline & Total de respuestas & 438 & \multicolumn{3}{|c|}{ Total de respuestas } \\
\hline \multicolumn{3}{|c|}{ Agencias de Evaluación: ANECA, ANEP, CNEAI } & \multicolumn{3}{|c|}{ Calidad de Congresos y participación } \\
\hline Respuesta & Porcentaje & Cantidad & $\overline{\text { Respuesta }}$ & $\begin{array}{l}\text { Porcentaje } \\
\end{array}$ & Cantidad \\
\hline-3 & $27.92 \%$ & 122 & -3 & $7.82 \%$ & 34 \\
\hline-2 & 13.04\% & 57 & -2 & $8.51 \%$ & 37 \\
\hline-1 & 10.98\% & 48 & -1 & $10.57 \%$ & 46 \\
\hline 0 & $21.51 \%$ & 94 & 0 & $21.61 \%$ & 94 \\
\hline+1 & ${ }^{15.33 \%} \square$ & 67 & +1 & ${ }^{28.51 \%}$ & 124 \\
\hline+2 & $824 \%$ & 36 & +2 & $18.16 \%$ & 79 \\
\hline \multirow[t]{2}{*}{+3} & $2.97 \%$ & 13 & +3 & ${ }_{4.83 \%}$ & 21 \\
\hline & Total de respuestas & 437 & & & 435 \\
\hline
\end{tabular}

Fuente Proyecto MapCom

http://www.mapcom.es/media/files/Los_investigadores_de_la_comunicacion_en_Espana_ante_el_espejo.

Encuesta_MapCom.pdf

Tabla 5. Debilidades y fortalezas de la Investigación en Comunicación, al valorar las MARCOS INSTITUCIONALES de Congresos y la Indexación de revistas científicas.

\begin{tabular}{|c|c|c|c|c|c|}
\hline \multicolumn{3}{|c|}{ Calidad de Congresos y participación } & \multicolumn{3}{|c|}{ Indexación de revistas científicas } \\
\hline$\overline{R e s p u e s t a}$ & Porcentaje & Cantidad & Respuesta & Porcentaje & Cantidad \\
\hline-3 & $7.82 \%$ & 34 & -3 & $14.87 \%$ & 65 \\
\hline-2 & $8.51 \%$ & 37 & -2 & $6.64 \%$ & 29 \\
\hline-1 & $10.57 \%$ & 46 & -1 & $9.84 \%$ & 43 \\
\hline 0 & $21.61 \%$ & 94 & 0 & $18.76 \%$ & 82 \\
\hline+1 & $28.51 \% \square$ & 124 & +1 & $23.57 \%$ & 103 \\
\hline+2 & $18.16 \%$ & 79 & +2 & $18.31 \%$ & 80 \\
\hline+3 & $4.83 \%$ & 21 & +3 & $801 \%$ & 35 \\
\hline & Total de respuestas & 435 & & Total de respuestas & 437 \\
\hline
\end{tabular}

Fuente Proyecto MapCom

http://www.mapcom.es/media/files/Los_investigadores_de_la_comunicacion_en_Espana_ante_el_espejo.

Encuesta_MapCom.pdf

Los perfiles de la valoración que atañen a la Calidad de congresos y su participación, y a la Indexación de las revistas científicas, no suponen juzgar condiciones de los procesos de investigación, sino valorar las valoraciones de sus resultados, es decir, meta-valorar los marcos de su reconocimiento institucional. Y en este caso, esta meta-valoración aparece muy mayoritariamente positiva. Así pues, dado que, 
como expusimos al principio, la muestra obtenida por el retorno de respuestas a la encuesta permite representarse significativamente al universo los investigadores censados en centros la universidad española con docencia reglada en Comunicación, se podría concluir que estos son menos críticos ante la meta-valoración de su actividad investigadora que frente a las condiciones materiales y organizacionales en que la desarrollan. ¿Y cómo este censo de investigadores encaja dentro de la población universitaria cuando se retienen rasgos de edad, género, formación de origen e ingresos económicos? Terminaremos este informe con la exposición de tales datos.

\section{b) La valoración de la experiencia investigadora en relación al beneficio obtenido como retorno}

A la hora de valorar la propia experiencia investigadora referida al beneficio obtenido como retorno, primero se preguntó por las revistas indexadas y no indexadas en CC. Sociales. La Tabla 6 permite comparar los datos sobre las valoraciones alternativas atribuidas por los encuestados a las revistas indexadas y no indexadas; a esta pregunta contestaron entre 370 y 390 encuestados.

Los datos son reveladores. A las revistas se le atribuyen mayor influencia que a los libros y monografías y que a las Actas de los congresos (Tabla 7), sobre todo por los criterios de marcos financieros y de elección de objetos y campos de estudio, en primer lugar, y por los criterios de contextualización teórica, en segundo lugar, puesto que este criterio sobresale cuando es valorado "no demasiado".

Las ediciones de libros y monografías, así como las Actas de Congresos, resultan menos valorados, pero especialmente reteniendo los criterios de Elección de objetos y campos de estudio y de Elección de métodos y técnicas. Así pues, por una parte se constata una visión crítica sobre rendimientos de la investigación en lo que atañe a la publicación de resultados, de los cuales se valoran menos sus aplicaciones que el marco financiero que los sustenta; por otra parte, si se trata de las revistas de mayor impacto, se constata la elección de objetos y campos de estudio. Así pues, por una parte se constata una visión crítica sobre rendimientos de la investigación en lo que atañe a la publicación de resultados, de los cuales se valoran menos sus aplicaciones que el marco financiero que los sustenta; por otra parte, si se trata de las revistas de mayor impacto, se constata especialmente la elección de objetos y campos de estudio.

También al objeto de valorar la propia experiencia investigadora referida al beneficio obtenido como retorno, se preguntó en segundo lugar por la dimensión del retorno o el rendimiento dominante en función de los objetivos que la investigación ha perseguido. La Tabla 8 permite comparar los datos sobre los diferentes rendimientos dominantes obtenidos en relación a los objetivos perseguidos por la actividad investigadora. Las publicaciones y ediciones vuelven a ser citadas como rendimiento mayoritariamente dominante en general, pero sobre todo para los objetivos de "explicar" y "evaluar", así como algo menos citadas la presentación en congresos. El retorno por divulgación/formación ocupa un tercer rango.

Se puede resaltar también que el total de respuestas a esta pregunta es notablemente inferior que el número de respuestas a las preguntas inmediatamente anteriores, pues el número de encuestados que la responden oscila entre un mínimo de 284 y un máximo de 364. 
Tabla 6. Valoración sobre cuánto influyen en la investigación los criterios de publicación en las revistas indexadas y no indexadas.

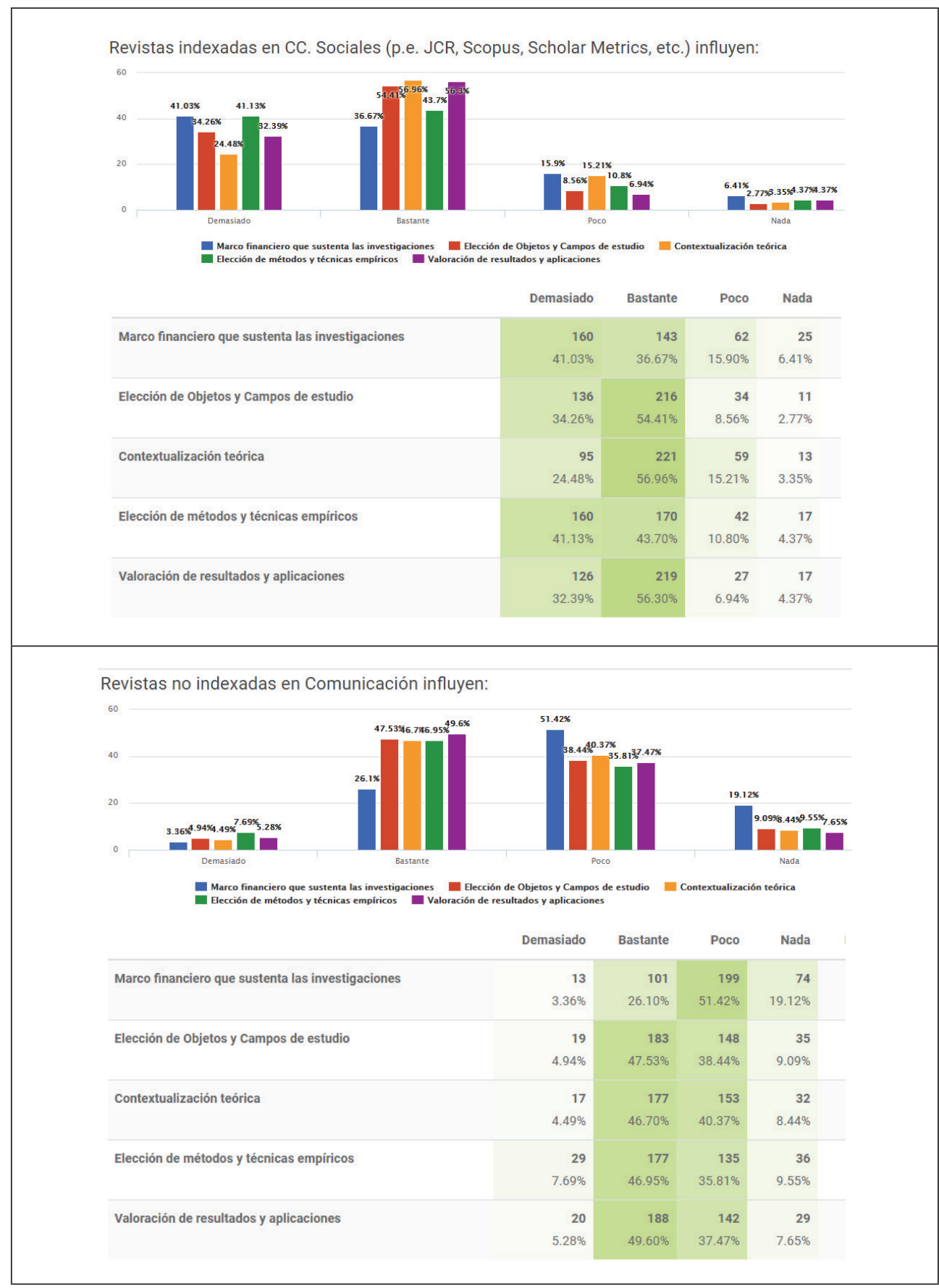

Fuente Proyecto MapCom

http://www.mapcom.es/media/files/Los_investigadores_de_la_comunicacion_en_Espana_ante_el_espejo. Encuesta_MapCom.pdf 
Tabla 7. Valoración sobre cuánto influyen en la investigación los criterios de publicación en las ediciones de librpos y monografías y en Actas de Congresos.

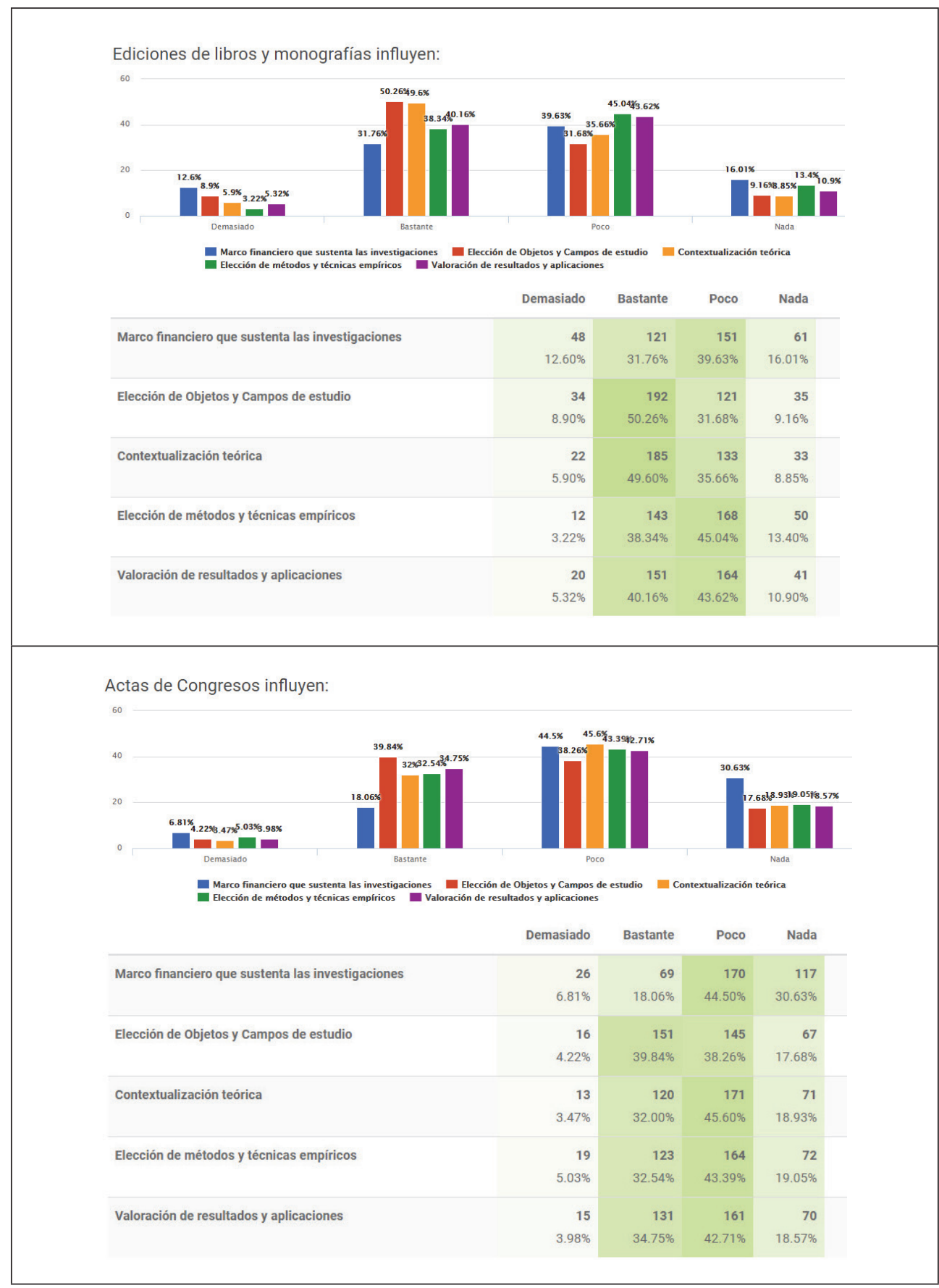

Fuente Proyecto MapCom

http://www.mapcom.es/media/files/Los_investigadores_de_la_comunicacion_en_Espana_ante_el_espejo. Encuesta_MapCom.pdf 
Tabla 8. Dimensión del retorno o el rendimiento dominante en función de los objetivos que la investigación ha perseguido.

\begin{tabular}{|c|c|c|c|c|c|c|c|c|}
\hline OBJETIVOS & $\begin{array}{l}\text { Presentación/ } \\
\text { congresos }\end{array}$ & $\begin{array}{c}\text { Publicación/ } \\
\text { ediciones }\end{array}$ & $\begin{array}{l}\text { Open } \\
\text { Access }\end{array}$ & $\begin{array}{l}\text { Citas/ } \\
\text { reseñas }\end{array}$ & $\begin{array}{c}\text { Divulgación/ } \\
\text { formación }\end{array}$ & $\begin{array}{c}\text { Aplicaciones } \\
\text { técnicas y } \\
\text { sociales }\end{array}$ & $\begin{array}{c}\text { Sin } \\
\text { retornos }\end{array}$ & $\begin{array}{l}\text { Total de } \\
\text { respuestas }\end{array}$ \\
\hline $\begin{array}{l}\text { DESCRIBIR: registrar, clasificar, } \\
\text { catalogar, presentar y/o def inir } \\
\text { prácticas de comunicación para, p. } \\
\text { e., medir audiencias, cuantif icar } \\
\text { resultados, etc. }\end{array}$ & $\begin{array}{c}127 \\
36.49 \%\end{array}$ & $\begin{array}{c}152 \\
43.68 \%\end{array}$ & $\begin{array}{c}13 \\
3.74 \%\end{array}$ & $\begin{array}{c}13 \\
3.74 \%\end{array}$ & $\begin{array}{c}25 \\
7.18 \%\end{array}$ & $\begin{array}{c}7 \\
2.01 \%\end{array}$ & $\begin{array}{c}11 \\
3.16 \%\end{array}$ & 348 \\
\hline $\begin{array}{l}\text { EXPLICAR: establecer relaciones } \\
\text { entre características del objeto de } \\
\text { estudio proponiendo causas, ef } \\
\text { ectos, correlaciones y/0 evolución } \\
\text { del objeto de estudio, recurriendo, } \\
\text { a teorías conocidas, o planteando } \\
\text { alguna nueva. }\end{array}$ & $\begin{array}{c}52 \\
14.29 \%\end{array}$ & $\begin{array}{c}239 \\
65.66 \%\end{array}$ & $\begin{array}{c}11 \\
3.02 \%\end{array}$ & $\begin{array}{c}15 \\
4.12 \%\end{array}$ & $\begin{array}{c}29 \\
7.97 \%\end{array}$ & $\begin{array}{c}7 \\
1.92 \%\end{array}$ & $\begin{array}{c}11 \\
3.02 \%\end{array}$ & 364 \\
\hline $\begin{array}{l}\text { EVALUAR o valorar: poner } \\
\text { a prueba, contrastar o validar } \\
\text { modelos. Así, p. e., refutar o conf } \\
\text { irmar la reducción de disonancias } \\
\text { cognitivas cuando se estudian los } \\
\text { cambios de actitudes, o contrastar } \\
\text { por análisis del framing los } \\
\text { discursos nacionalistas. }\end{array}$ & $\begin{array}{c}64 \\
20.92 \%\end{array}$ & $\begin{array}{c}148 \\
48.37 \%\end{array}$ & $\begin{array}{c}13 \\
4.25 \%\end{array}$ & $\begin{array}{c}17 \\
5.56 \%\end{array}$ & $\begin{array}{c}27 \\
8.82 \%\end{array}$ & $\begin{array}{c}17 \\
5.56 \%\end{array}$ & $\begin{array}{c}20 \\
6.54 \%\end{array}$ & 306 \\
\hline $\begin{array}{l}\text { INTERVENIR: utilizar modelos } \\
\text { conocidos para cambiar conductas } \\
\text { o procesos sociales. Así, p. } \\
\text { e., se hace en la investigación } \\
\text { aplicada o en la investigación/ } \\
\text { acción, como sucede si se llevan } \\
\text { a cabo dinámicas de grupos para } \\
\text { modificación de conducta o si se } \\
\text { aplica la técnica del Brainstorming } \\
\text { para incrementar la creatividad. }\end{array}$ & $14.44 \%$ & $27.82 \%$ & $3.17 \%$ & $2.46 \%$ & $\begin{array}{c}51 \\
17.96 \%\end{array}$ & $\begin{array}{c}51 \\
17.96 \%\end{array}$ & $\begin{array}{c}46 \\
16.20 \%\end{array}$ & 284 \\
\hline
\end{tabular}

Fuente Proyecto MapCom

http://www.mapcom.es/media/files/Los_investigadores_de_la_comunicacion_en_Espana_ante_el_espejo. Encuesta_MapCom.pdf

Otro de los rendimientos de la experiencia investigadora, por los que se les preguntó a los encuestados, tenía que ser referido a los rendimientos que sus publicaciones e impactos de investigación produjeron en sus trayectorias profesionales, detallando "Contratos para empresas o instituciones", "Movilidad: estancias y becas", "Evaluaciones académicas: sexenios", y "Acreditación académica: Doctorado, Titularidad, Cátedra, etc.”. En la Tabla 9 se ofrecen los gráficos con las valoraciones expresadas por los encuestados sobre la importancia atribuida a tales rendimientos. 
A la vista de la comparación de los datos en la valoración de tales rendimientos en la trayectoria profesional de los encuestados, aparece que mayoritariamente se valoran como "nada importante" las contrataciones externas al ámbito universitario, así como las evaluaciones académicas referidas a los sexenios, mientras que las acreditaciones académicas, por el contrario, aparecen mayoritariamente valoradas como rendimiento "muy importante", y que la movilidad académica subvencionada es vista también por una mayoría simple como rendimiento "bastante importante" en la trayectoria profesional. Este es otro aspecto por el cual se viene a corroborar también que en las trayectorias profesionales la vida universitaria y la vida socioeconómica externa se dan la espalda, y que los rendimientos más valorados de la experiencia investigadora están orientados a la movilidad esencialmente académica.

\section{Discusión y Conclusiones}

Hasta aquí se han expuesto y comentado con mayor o menor detalle los perfiles de las respuestas de los investigadores censados en centros universitarios españoles con docencia reglada en Grado y Posgrado en Comunicación, invitados a cumplimentar un cuestionario que aspiraba a sondear las experiencias de su actividad investigadora reciente. Fueron invitados por correo electrónico a abrir el formulario en línea mediante un enlace y una contraseña, 2418 doctores censados como docentes en aquellos centros universitarios, más un número indefinido de doctores sin docencia, miembros de sociedades científicas, y de doctorandos inscritos en Programas de Doctorado, a los que respectivamente cursaron la invitación los responsables de las sociedades científicas y los coordinadores de los Programas de Doctorado.

Tabla 9. Rendimientos de impactos de investigación sobre trayectorias profesionales.

\begin{tabular}{|c|c|}
\hline Contratos empresas o instituciones & Movilidad: estancias y becas \\
\hline Evaluaciones académicas: sexenios & Acreditación académica: Doctorado, Titularidad, \\
Cátedra, etc.
\end{tabular}

Fuente Proyecto MapCom http://www.mapcom.es/media/files/Los_investigadores_de_la_comunicacion_en_Espana_ante_el_espejo. Encuesta_MapCom.pdf 
Respondieron total o parcialmente la encuesta 838 investigadores, que representaban un $34,66 \%$ del censo, y hecha la medida de la representatividad de esta muestra respecto al censo, se comprobó que lo era por los estratos de Universidad y Comunidad Autónoma. A este número de investigadores del censo cuyas respuestas han sido tratadas y comentadas (838), se puede añadir como dato a destacar que contestaron la encuesta hasta el final 496, mientras que el resto (342), no la completaron. Por otra parte, es también indicativo que, del universo de los 2418 que recibió la invitación, 416 la abrieron y la abandonaron acto seguido sin respuesta a ninguna pregunta.

Las líneas de investigación, la fijación de objetivos, la elección de objetos de estudio, y el tipo de técnicas empleadas para elaborar e interpretar datos, es una información que permite, por otra parte, aproximarse a las representaciones que los propios investigadores tienen de su actividad, determinando con ello el producto de la investigación. Pues bien, a este propósito conviene resaltar que la evaluación de la calidad investigadora es valorada no tanto por la excelencia de sus resultados, por el avance de conocimiento, y por retornos sociales, cuanto por el impacto de las publicaciones conforme al número de citas entre las élites de investigadores, mostrándose así más una competencia corporativa entre ellos, que una competencia científica por el conocimiento y sus aplicaciones.

De los 479 de los 838 encuestados (el 57,2\%) que brindaron palabras clave para describir líneas de investigación se siguió como resumen la formulación de los siguientes campos de estudio: Comunicación Mediática, Comunicación organizacional, Comunicación de Grupos, Comunicación interpersonal, Teoría y Epistemología y Métodos y técnicas. Y fue la comunicación mediática la que destacó sobre las demás como campo de estudio. Hay dos condociones a señalar para esta dominancia: desde su origen nuestras facultades universitarias se orientaron a cumplir la función social de la formación superior de profesionales para los Medios, sin orientarse a la formación de profesionales en otras prácticas sociales de la comunicación, como puede ser la educación, la sanidad, etc. y, hoy día, por otra parte, la emergencia de las prácticas mediadoras del universo digital vienen a sobre-representar este perfil en la elección de campos de estudio. Dada la prioridad del objeto de estudio integrado en el campo de la Comunicación mediática, no es de extrañar, entonces, que las técnicas más utilizadas sean las de análisis documentales (Análisis de contenido, Análisis del discurso, Análisis de documentación).

Los encuestados comparten similar frecuencia en la elección de objetivos de investigación sobre objetos de estudio mayoritariamente del campo de la Comunicación mediática, siendo el más frecuente de estos objetivos el de "Describir" y el menos frecuente el de "Intervenir", lo que es indicador de que asistimos a los primeros desarrollos de una disciplina, más que al desarrollo característico de su madurez, lo que se traduciría en un mayor número de investigaciones centradas en aplicaciones sociales.

Estas apreciaciones que acaban de expresarse, vienen confirmadas por las representaciones a propósito de la valoración de la experiencia investigadora en relación con el beneficio obtenido como retorno. Y en este sentido, en primer lugar, a las revistas se le atribuyen en la valoración de retorno mayor influencia que a los libros y monografías y que a las Actas de los congresos, confirmándose así una vez más que la competencia corporativa entre investigadores se impone sobre la competencia científica por el conocimiento y sus aplicaciones. Así pues, aunque se constata una visión crítica sobre rendimientos de la investigación en lo que atañe a la publicación 
de resultados, se valoran menos las aplicaciones de éstos, que el marco financiero que los sustenta, o si se trata de las revistas de mayor impacto, que la elección de objetos y campos de estudio, especialmente.

Redundando en la valoración de las publicaciones científicas, estas vuelven a ser citadas como rendimiento mayoritariamente dominante en general, pero sobre todo para los objetivos de "Explicar" y "Evaluar", función diagnóstica en detrimento de la función de intervención social. Se posterga incluso al retorno por divulgación/ formación, que ocupa un tercer rango.

En las trayectorias profesionales, la vida universitaria y la vida socioeconómica externa se dan la espalda. Se valoran como "nada importante" las contrataciones externas al ámbito universitario, así como, seguramente por despecho, las evaluaciones académicas referidas a los sexenios. La percepción entre los investigadores es que los rendimientos más valorados de la experiencia investigadora son las acreditaciones académicas, seguidas de la movilidad académica, lo cual confirma igualmente la competencia corporativa.

Como también forma parte de las representaciones de la propia actividad investigadora la valoración del esfuerzo que se invierte en ella, se preguntó sobre si este esfuerzo se producía aprovechando las condiciones positivas o si se invertía enfrentando condiciones negativas. Se preguntó así, en primer lugar, por la valoración positiva o negativa de las condiciones materiales: subvenciones y financiación, becas y contratos, trámites burocráticos, e Instalaciones y laboratorios); en segundo lugar por la valoración positiva o negativa de las condiciones organizacionales: requisitos exigidos en convocatorias, programas de Formación metodológica, redes de cooperación y conocimiento, y recursos humanos en los equipos; y en tercer lugar, por la valoración positiva o negativa de las condiciones institucionales de los marcos en que se desarrollan los procesos de investigación: compatibilidad investigación y Docencia, relaciones entre Universidad y Empresa, Agencias de Evaluación, Calidad de Congresos y participación e Indexación de revistas científicas. Pues bien, las condiciones materiales que acompañan los procesos de investigación son valoradas negativamente, salvo al referirse a instalaciones y laboratorios, cuya valoración es en conjunto positiva. En cambio, domina la valoración positiva a propósito de las condiciones organizacionales de los procesos de investigación, si bien los requisitos exigidos en convocatorias públicas y los programas de formación metodológica son valorados más negativamente que las redes de cooperación y conocimiento, y los recursos humanos en los equipos. Finalmente, al apreciar las condiciones de los marcos institucionales, aparecen mayoritariamente valoraciones positivas sólo al juzgar la calidad de Congresos y participación, mientras que son más frecuentes las valoraciones extremadamente negativas (-3) al juzgar la compatibilidad entre investigación y docencia, las relaciones entre Universidad y Empresa y al juzgar a las Agencias de evaluación ANECA, ANEP, y CNEAI.

Por último, con esta encuesta, que es la primera vez que se aplica a un censo de investigadores tan amplio en un país, y de una especialidad tan concreta como esta, hemos podido aproximarnos a sus perfiles de encuadre en su actividad investigadora y a sus representaciones sobre la propia experiencia y actividad en curso en lo que atañe a sus valoraciones sobre condiciones y rendimiento de la investigación, gracias a lo cual se ha podido descubrir el capital humano que lo integra, sus fortalezas y debilidades, al objeto de poder desarrollar las políticas científicas más adecuadas a su potencial. 


\section{Bibliografía}

Arcila, C.; Piñuel, J. L.; Calderin, M. (2013). The e-Research on Media \& Communications: Attitudes, Tools and Practices in Latin America Researchers. [La e-investigación de la Comunicación: actitudes, herramientas y prácticas en investigadores iberoamericanos]. Comunicar, 40, 111-118. https://doi.org/10.3916/C40-2013-03-01

Barranquero, A.; Limön, N. (2017): “Objetos y métodos dominantes en comunicación para el desarrollo y el cambio social en las Tesis y Proyectos de Investigación en España (20072013)". Revista Latina de Comunicación Social, 72, pp. 1 a 25. http://www.revistalatinacs.org/072paper/1151/01es.html DOI: 10.4185/RLCS-2017-1151

Blázquez, M. (2015): Tesis Doctorales en las Universidades Españolas durante el periodo 1977-2014. En: http://mblazquez.es/tesis-doctorales-en-las-universidades-espanolas-durante-el-periodo-1977-2014/.

Caffarel, C.; Caceres, D. (1993). "La comunicación en España: planteamientos temáticos y metodológicos entre 1987 y 1990. La investigación en España”. Cuadernos de investigación en comunicación (Cinco), n. 29, pp. 23-30.

Caffarel, C.; Domínguez, M.; Romano, V. (1989). "El estado de la investigación en comunicación en España (1978-1987)". Cuadernos de investigación en comunicación (Cinco), n. 3, pp. 45-57.

Caffarel C.; Gaitán, J. A.; Lozano, C.; Piñuel, J. L. (2018). “La investigación en comunicación en España: un problema para sus investigadores” en Aarón Rodríguez y Samuel Gil Soldevilla, Investigar en la era neoliberal. Visiones críticas sobre la investigación en comunicación en España. Barcelona, Bellaterra, Castellón y Valencia: Colección Aldea Global (UAB, UPF, UJI, UV)

Caffarel, C., Izquierdo, P.; Núñez, S. (2018). ¿Cómo investiga la mujer cuando investiga sobre la mujer en comunicación? En Estrella Martínez (Coord.) Propuestas de investigación en áreas de vanguardia. Madrid: Tecnos. Pp. 73-86

Caffarel, C.; Ortega, F.; Gaitán, J. A: (2017). Investigación en Comunicación en la Universidad Española en el periodo 2007-2014. El profesional de la información. vol. 26, n. 2, pp. 218-227

Castillo, A.; Xifra, J. (2006). Investigación bibliométrica de las tesis doctorales españolas sobre relaciones públicas (1965-2005). Anàlisi 34, 141-161.

Delgado-López-Cózar, E.; Torres-Salinas, D.; Jiménez-Contreras, E.; Ruiz-Pérez, R. (2006). "Análisis bibliométrico y de redes sociales aplicado a las tesis bibliométricas defendidas en España (1976-2002): temas, escuelas científicas y redes académicas”. Revista española de documentación cientifica, V. 29, N. 4, PP. 493- 524.

Díaz Nosty, B.; De Frutos, R. (Coord.) (2016). Rumbos de la Comunicación en España. III Congreso Nacional sobre Metodología de la Investigación en Comunicación, Málaga, 12 y 13 de noviembre de 2015.

Férnandez, D.; Masip, P. (2013): Tres décadas de investigación española en comunicación: hacia la mayoría de edad, [Three Decades of Spanish Communication Research: Towards Legal Age] Comunicar, 41, 15-24. doi: http://dx.doi.org/10.3916/C41-201301

Fuentes, E.; Arguimbau, L. (2010). Las tesis doctorales en España (1997-2008): análisis, estadísticas y repositorios cooperativos. Revista Española de Documentación Cientifica, 33(I), 63-89. doi: http://dx.doi.org/10.3989/redc.2010.1.711

Gaitán Moya, J. A.; Lozano Ascencio, C.; Piñuel Raigada, J. L. (2016). "Prospectiva de la investigación sobre TV a partir de los Proyectos I+D y Tesis doctorales de 2007 a 2013 
en las Facultades españolas de comunicación”. Revista de la Asociación Española de Comunicación, Vol. 6, № 3, pp.51-59.

Gaitan, J. A.; Lozano, C.; Piñuel, J. L. (2016) "Prospectiva de la investigación sobre TV a partir de los Proyectos I+D y Tesis doctorales de 2007 a 2013 en las Facultades españolas de comunicación". Revista de la Asociación Española de Investigación de la Comunicación (RAEIC). Volumen 3. No 6. Segundo semestre. pp. 51 -59.

Jones, D. E. (1998). "Investigación sobre comunicación en España. Evolución y perspectivas". Zer, n. 5, pp. 13-51.

Jones, D. E. (1994). Investigació sobre comunicació al'Espanya dels noranta. Centre d'Investigació de la Comunicació i Universitat Autònoma de Barcelona. Cultura y comunicación social: América Latina y Europa Ibérica. Barcelona: Generalitat de Catalunya.

Jones, D. E.; Baró, J.; Landa, C.; Ontalba-Ruipérez, J. A. (2000). Investigación sobre comunicación en España: aproximación bibliométrica a las tesis doctorales (1926-1998). Barcelona: ComCat.

López, P.; Vicente, M. (2011): Métodos y técnicas de investigación dominantes en las revistas científicas españolas sobre comunicación (2000-2009). En José-Luis Piñuel, Carlos Lozano \& Alberto García (Eds.): Investigar la comunicación en España, (pp. 665-679). Madrid: AE-IC/URJC.

López_Escobar, E.; Martín Algarra, M. (2016). Communication teaching and research in Spain The calm and the storm, Publizistik (2017) 62:83-105, DOI 10.1007/s11616-0160306-4

Lozano Ascencio, C.; Piñuel Raigada, J. L. (2015). MAPIBERCOM. Una metodología para configurar un Mapa interactivo de Investigación en Iberoamérica sobre prácticas sociales de Comunicación. XIV Congreso Internacional IBERCOM 2015 Universidad de Sao Paulo. Brasil. 3-13 pp.

Lozano, C.; Gaitán, J. A. (2011). Dedicación a la investigación y a la docencia universitaria en comunicación: compatibilidad y perfiles en España, Europa y América Latina. En Piñuel, José-Luis; Lozano, Carlos \& Alberto García (Eds.). Investigar la Comunicación en España (pp.587-595). Fac. de CC. de la Comunicación. Fuenlabrada: Universidad Rey Juan Carlos.

Martín Algarra, M.; Serrano-Puche, J.; Rebolledo, M. (2018). La mujer en la investigación en comunicación en España: un análisis de la producción científica (2007-2013). En: adComunica. Revista Científica de Estrategias, Tendencias e Innovación en Comunicación, $\mathrm{n}^{\mathrm{o}} 15$. 65-87. DOI: http://dx.doi. org/10.6035/2174-0992.2018.15.5

Martínez, M. (2009). La investigación sobre comunicación en España. Evolución histórica y retos actuales. [The Communication Research in Spain. Historical evolution and current challenges]. Revista Latina de Comunicación Social, 64, 1-14. doi: http://dx.doi. org/10.4185/RLCS-64-2009-800-01-14

Mártinez, M.; Saperas, E. (2011). La investigación sobre Comunicación en España (19982007). Análisis de los artículos publicados en revistas científicas, Revista Latina de Comunicación Social, 66, 101-129. doi: http://dx.doi.org/10.4185/RLCS-66-2011-926-101129

Ortega, F.; Azurmendi, A.; Munöz Saldaña, M. (2018). Metodologías Avanzadas de Investigación en Comunicación y Ciencias Sociales, la revolución de los instrumentos y los métodos, Qualtrics, Big Data, Web Data et al. Tendencias metodológicas de las prácticas de investigación en comunicación en España. Salamanca: Comunicación Social

Peñafiel, C.; Torres, E.; Izquierdo, P., (2017) Percepción cualitativa de gestores universitarios de investigación en Comunicación a través de un Philips 66. En Herrrero, F. J.; Mateos, 
C. (coord.): Del verbo al bit, Universidad de la Laguna, págs. 790-810. Libro colectivo en línea: http:/www.revistalatinacs.org/16SLCS/libro-colectivo-edicion-2.html

Piñuel Raigada, J. L.; Lozano Ascencio, C.; García Jiménez, A. Eds. (2011) Investigar la comunicación en España. Vol. I. Universidad Rey Juan Carlos. Fuenlabrada (Madrid)

Piñuel, J. L.; Gaitán, J. A.; Lozano, C.; Gallardo, L. (2017) "Perfiles de la investigación en proyectos de $\mathrm{i}+\mathrm{d}$ y tesis doctorales en área I de MapCom” en Nosty, B y Frutos, R (Coords.) Tendencias de la Investigación Universitaria Española en Comunicación. Ed. Thomson Reuters pp. 35-58.

Piñuel, J. L.; Lozano, C.; Gaitán, J. A. (2015) "Propuesta de estudio para realizar un mapa de la investigación en comunicación en América Latina" Revista Latinoamericana de Ciencias de la Comunicación (ALAIC). v. 12, n. 22 (12). enero-junio. 2015 44-54 pp. ISSN 1807-3026.

Piñuel-Raigada, J. L. (2011). La docencia y la investigación universitarias en torno a la Comunicación como objeto de estudio en Europa y América Latina. Colección Cuadernos Artesanos de Latina, n. 15. La Laguna-Tenerife: SLCS.

Repiso, R.; Delgado, E.; Torres, D. (2011b). Análisis bibliométrico y de redes sociales en tesis doctorales españolas sobre televisión (1976/2007) [Bibliometric and Social Network Analysis Applied to Television Dissertations Presented in Spain (1976/2007)]. Comunicar, 37(XIX), 151-159. doi: http://dx.doi.org/10.3916/C37-2011-03-07.

Rodrigo-Alsina, M.; García-Jiménez, L. (2010). "Communication theory and research in Spain: A paradigmatic case of a socio-humanistic discipline". European journal of communication, v. 25, n. 3, pp. 273-286. 